Communications in Physics, Vol.31, No. 1 (2021), pp. 35-43

DOI:10.15625/0868-3166/15391

\title{
MOLECULAR EMISSIONS FROM CIRCUMSTELLAR ENVELOPES IN THE PRESENCE OF A BINARY COMPANION
}

\author{
TRAN NGOC HUNG ${ }^{1,2, \dagger}$, DINH VAN TRUNG ${ }^{1 \ddagger}$, PHAM DONG BANG $^{1,2}$ \\ AND NGUYEN THI THANH BAO ${ }^{1,2}$ \\ ${ }^{1}$ Institute of Physics, Vietnam Academy of Science and Technology, \\ 18 Hoang Quoc Viet, Cau Giay, Hanoi, Vietnam \\ ${ }^{2}$ Graduate University of Science and Technology, \\ Vietnam Academy of Science and Technology, 18 Hoang Quoc Viet, Cau Giay, Hanoi, Vietnam \\ E-mail: ${ }^{\dagger}$ tnhung@iop.vast.ac.vn; ${ }^{\ddagger}$ dvtrung@iop.vast.ac.vn
}

Received 18 June 2020

Accepted for publication 20 August 2020

Published 05 January 2021

\begin{abstract}
Following our previous work on the hydrodynamic simulations of the structure of circumstellar envelopes in the presence of a binary companion, in this paper we present the results of radiative transfer calculations for molecular emission line $\mathrm{HC}_{3} \mathrm{NJ}=5-4$ from these simulated circumstellar envelopes. We show that the molecular line emission traces closely the spiral pattern and the associated density enhancement induced by the presence of the binary companion. The molecular emission provides the spatial kinematics of the features within the envelope, which is valuable for estimating the orbital parameters of the binary system and for inferring the physical conditions of the gas within the envelope. We also show that the appearance of the molecular emission depends on the viewing angle resulting in a range of shapes from the spiral pattern to ring-like features, similar to that observed recently in a number of circumstellar envelopes at high angular resolution.
\end{abstract}

Keywords: circumsteller envelope; molecular emission.

Classification numbers: 79.10.Fy.

\section{INTRODUCTION}

The mass loss from intermediate mass stars in the asymptotic giant branch (AGB) phase through the dusty and dense stellar wind creates an expanding circumstellar envelope around the star. The envelope is generally expected to be roughly spherically symmetric due to the underlying radiation pressure on dust grains as the main mechanism for mass loss from AGB stars [1]. The expanding envelope of AGB star is the large laboratory containing dust, atomic and molecular (C)2021 Vietnam Academy of Science and Technology 
gases. As a result, the circumstellar envelope around AGB stars is the source of strong molecular emission and numerous molecular species have been detected toward a number of prominent envelopes. The structure of the envelope is determined mainly by the mass loss process through slow dusty wind from the AGB star, providing a record of past stellar activities, allowing us to look back in time and reconstruct stellar evolution during this short but important AGB phase. Recently it became clear that drastic departure from this simple picture of circumstellar envelopes is quite common, with the observations of bipolar morphology, disk or collimated outflows and especially the appearance of arcs, rings and spiral patterns in a number of circumstellar envelopes [2-4]. Although different mechanisms have been proposed, the presence of a binary companion $[5,6]$ is regarded as the most viable cause of the observed unusual morphological features. Several groups have carried out hydrodynamic simulations with different geometrical configurations to study the effect of the binary companion on the structure of the envelopes $[2-4,7,8]$. We emphasize here that in order to compare theoretical predictions with available high angular resolution observations of molecular emission, radiative transfer calculations have been considered. However, such radiative transfer modeling was performed in only a few particular cases [4,9] and a good knowledge of how the molecular emission changes with the properties of the binary system is still lacking.

In a previous paper [10] we have explored the effect of a binary companion on the structure of the circumstellar envelope, especially the appearance of spiral pattern and arc or ring-like features as a function of binary orbital configuration using high resolution hydrodynamic simulations. In the present paper, we perform radiative transfer calculations for molecular emission using our previous results of hydrodynamic simulations and study the dependence of molecular emission on the binary orbital configuration and the viewing angle of the observer. The predicted molecular emissions provide both spatial distribution and also the velocity information of the molecular gas inside the envelope and will allow for comparison with available high angular resolution observations.

\section{MODEL DESCRIPTION}

\section{II.1. Hydrodynamic simulations}

We have carried out hydrodynamic simulations using publicly available code PLUTO [11] to investigate the influence of a binary companion in different orbital configurations from circular to highly elongated orbits. We consider a simulation domain of $\sim 4800$ astronomical units in two representative cases: the companion star moving in (i) circular orbit and in (ii) elliptical orbit with eccentricity $e=0.5$ with orbital period of 325 years [10]. The wind velocity from the AGB star is set at $15.7 \mathrm{~km} / \mathrm{s}$ and the mass loss rate is $10^{-5}$ solar mass per year, which are typical for AGB stars with high mass loss rate. The output of hydrodynamic simulations consists of gas density, velocity and temperature data on a three dimensional Cartesian grid. More details could be found in our previous publication [10].

\section{II.2. Radiative transfer simulations}

In this paper we focus on the molecule $\mathrm{HC}_{3} \mathrm{~N}$, which is commonly found in the inner region of the circumstellar envelope around carbon rich evolved stars. In typical circumstellar envelopes, the low lying rotational levels of $\mathrm{HC}_{3} \mathrm{~N}$ are known to be quite easily excited through collisions with molecular hydrogen [12]. Therefore, for simplicity we assume that the $\mathrm{HC}_{3} \mathrm{~N}$ molecules are in local thermodynamic equilibrium (LTE) condition within the simulation domain of the envelope. 
Under the LTE approximation, the population distribution on different rotational energy levels is calculated according to the Boltzmann distribution:

$$
n_{J}=g_{J} e^{-E_{J} / k T} / Z(T),
$$

where $k$ is the Boltzmann's constant. $\mathrm{E}_{\mathrm{J}}, \mathrm{g}_{\mathrm{J}}$ and $\mathrm{n}_{\mathrm{J}}$ are the energy, statistical weight and the population of the rotational level $J$, respectively. $Z(T)$ is the partition function of the $\mathrm{HC}_{3} \mathrm{~N}$ molecule at temperature $T$. We take into account all rotational levels up to $J=21$, which corresponds to an energy of $100 \mathrm{~K}$ above the ground state. We also assume that the abundance of this molecule with respect to molecular hydrogen is $10^{-6}$, in the range found for well studied envelopes such as $\mathrm{CW}$ Leo [13] and CIT 6 [12].

The hydrodynamic simulations produce data cubes with molecular gas density, velocity and pressure. We convert the pressure to temperature using usual ideal gas relation $P=n k T$ where $\mathrm{n}$ is the gas number density, $T$ is the gas temperature and $P$ is the gas pressure. Another important parameter of the model is the local linewidth of the emission line. We use the local linewidth at any point in the envelope as a combination of the usual thermal line width and a non-thermal component due to turbulent motion assumed to be $1 \mathrm{~km} / \mathrm{s}$.

In this paper we focus on the low lying rotational transition $J=5-4$ because the transition is most likely to be in LTE condition and optically thin, therefore avoiding the complication associated with optical depth effect [12]. For each viewing angle defined as the angle between the line of sight and the normal to the orbital plane of the binary system, the radiative transfer equation for transition $J=5-4$ is integrated along different parallel rays through the envelope. The output is a data cube containing the spectrum of brightness temperature for each projected position of the envelope on the plane of the sky.

\section{RESULTS AND DISCUSSION}

Figures 1, 2 and 3 showed the velocity channel maps of $\mathrm{HC}_{3} \mathrm{~N} J=5-4$ emission for the case of binary companion moving in a circular orbit at viewing angle of 0,50 and 90 degrees. The channel maps of the line emission exhibit the usual behavior of an expanding circumstellar envelope having the largest spatial extent at the systemic velocity of $0 \mathrm{~km} / \mathrm{s}$ and progressively becoming more compact at redshifted and blueshifted velocity channels. At 0 degree viewing angle, we can see that in nearly all velocity channels the spiral pattern is clearly shown with high contrast with only very weak emission in the inter-arm region. This is consistent with the results shown in our previous paper [10] that due to shock between fast and more slowly moving stellar wind, the gas density and temperature in the spiral structure are significantly higher (up to a factor of 10 for the gas density and a factor of 2 to 3 for the gas temperature) than in the inter-arm regions, leading to a strong enhancement of molecular emission. At the intermediate viewing angle of 50 degrees, the spiral pattern traced by molecular line emission becomes deformed and elongated. In high redshifted and blueshifted velocity channels, especially near the expansion velocity of $15.7 \mathrm{~km} / \mathrm{s}$ of the envelope, this effect is more pronounced and the spiral pattern is broken into arcs or incomplete rings. At viewing angle of 90 degrees, i.e. the observer is located in the orbital plane of the binary system, as shown in Fig. 3, only the structure out of the orbital plane of the spiral structure appears in the line emission. Near the systemic velocity one can see a series of alternating arcs extending almost to the polar axis of the envelope. This is one of the 

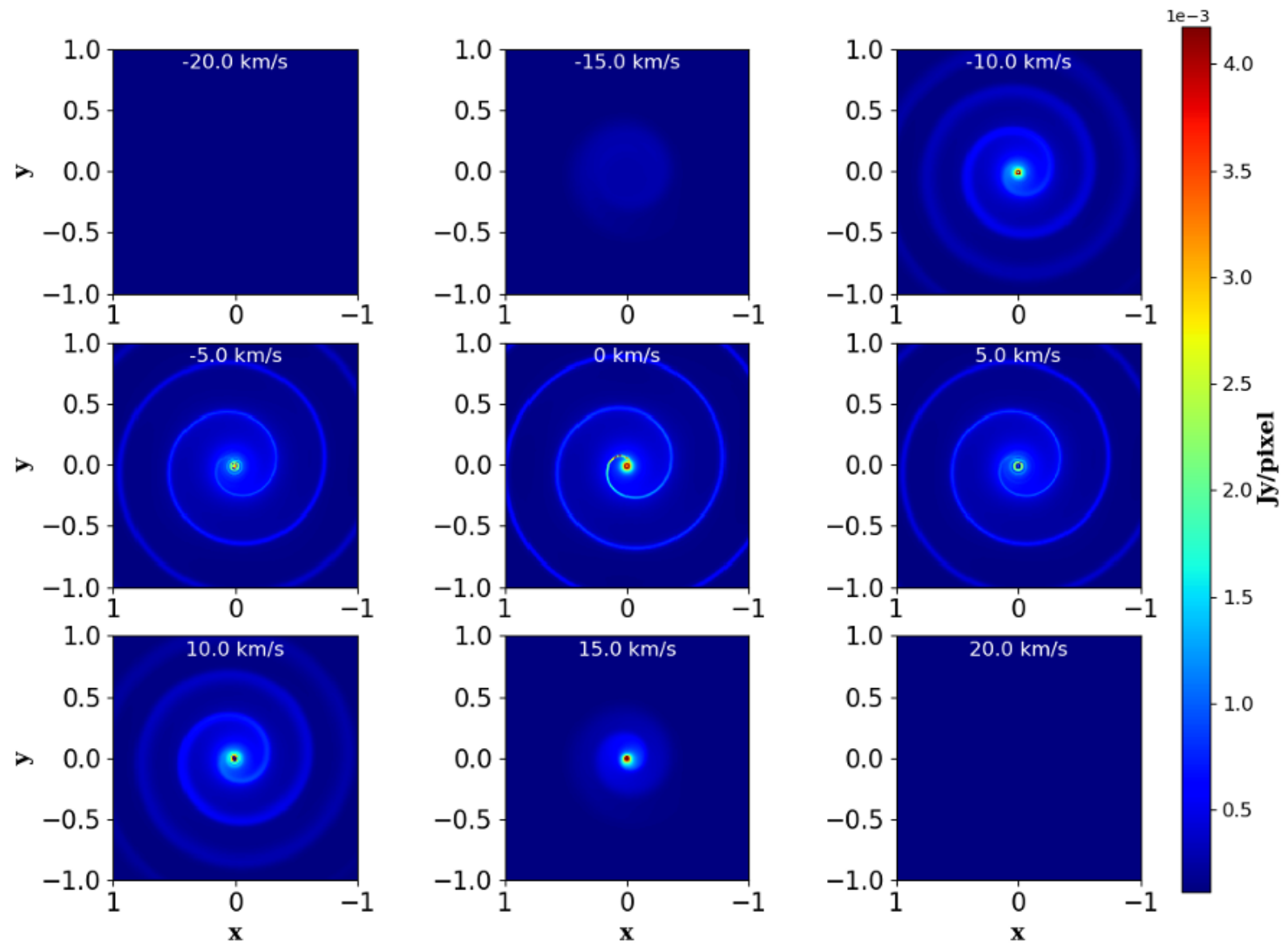

Fig. 1. Velocity channel maps of $\mathrm{HC}_{3} \mathrm{~N} \mathrm{~J}=5-4$ emission at viewing angle 0 degree for the case of binary companion moving in circular orbit around the AGB star. For presentation purpose the simulation domain is normalised so that the spatial extent of the envelope ranges from -1 to 1 in both directions on the plane of the sky.

features long thought to be characteristic for the presence of a binary companion [6,7] but has never been actually observed. In redshifted and blueshifted velocity channels these arcs deform and reconnect into ring-like features. However, the center of these rings is noticeably displaced from the position of the central mass losing AGB star. This unusual behaviour has been noticed in the case of IRC+10216 envelope where the arcs are off-centered with respect to the position of the AGB star [14]. There is a slight asymmetry intensity between redshifted and blueshifted velocity channels, most easily seen in the velocity channel $15 \mathrm{~km} / \mathrm{s}$ and $15 \mathrm{~km} / \mathrm{s}$ of the figures 1-3. That is a well known effect due to the optical depth of the $\mathrm{HC}_{3} \mathrm{~N} J=5-4$ line, resulting in the re-absorption of emission from the inner and hotter $\mathrm{HC} 3 \mathrm{~N}$ molecules by the outer and cooler gas in the approaching (blueshifted) part of the expanding circumstellar envelope [15].

As shown in our previous paper [10], changing the orbital geometry of the binary system from circular orbit to elliptical orbit, with increasing eccentricity $e$, new features in the spiral structure appears such as spur or bifurcation connecting the spiral arms. In addition, the shape of the spiral pattern becomes less regular and shows abrupt turn due to the accelerated orbital motion 

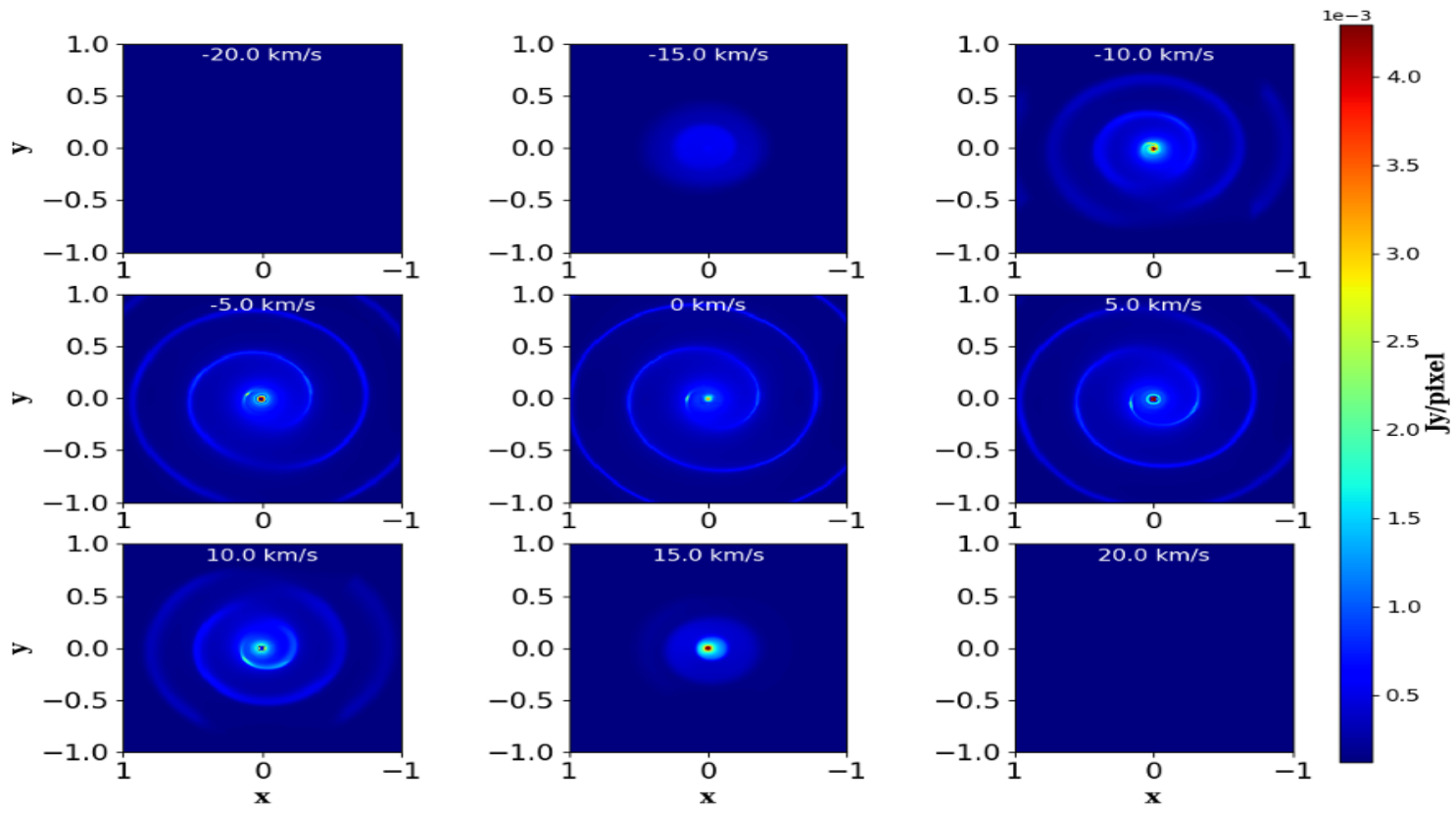

Fig. 2. Same as Figure 1 but for a viewing angle of 50 degrees.
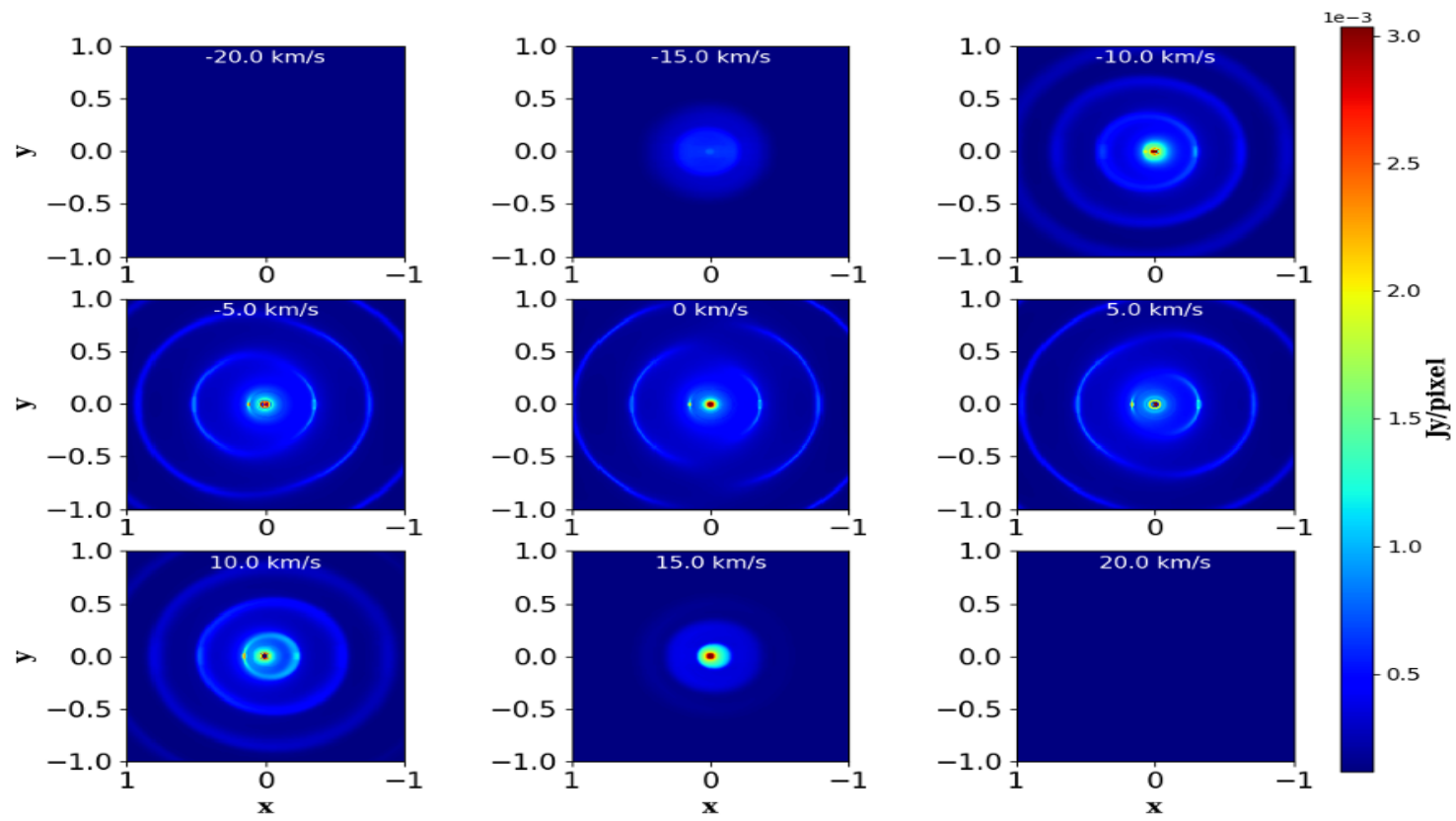

Fig. 3. Same as Fig. 1 but for a viewing angle of 90 degrees, i.e. the observer is located within the orbital plane of the binary system. 
of the companion and consequently the strong reflex motion of the AGB star during the passage around periastron. Figures 4, 5 and 6 clearly show that $\mathrm{HC}_{3} \mathrm{~N} \mathrm{~J}=5-4$ line emission exhibits nicely these features for the elliptical orbit with eccentricity e $=0.5$. Furthermore, the material bridge between spiral arms leads to a noticeable reduction of emission line intensity contrast between spiral arms and the inter-arm region as more molecular gas is piled up in the inter-arm region. As the viewing angle changes from 0 to 90 degrees, the molecular line emission morphs into a series of nearly concentric rings or arcs but offset from the position of the AGB star (see Figs. 5, 6). This interesting property of the spiral pattern induced by the presence of a binary companion in highly elliptical orbit has been remarked early $[7,8]$ and could help to explain the presence of concentric but offset arcs observed at high angular resolution by millimeter interferometric telescopes in some circumstellar envelopes such as IRC+10216 $[14,16]$. The change in morphology of the envelope might be more clearly appreciated in the angle - radius plot of the systemic velocity channel shown in Fig. 7. In the case of circular orbit, each winding of the spiral pattern is translated into a constant slope line with a quasi-sinusoidal variation. However, the binary companion moving in an elliptical orbit with $e=0.5$ induces a large variation near angle 2.0 radians with a band of fainter emission due to the bifurcation of the spiral arms.
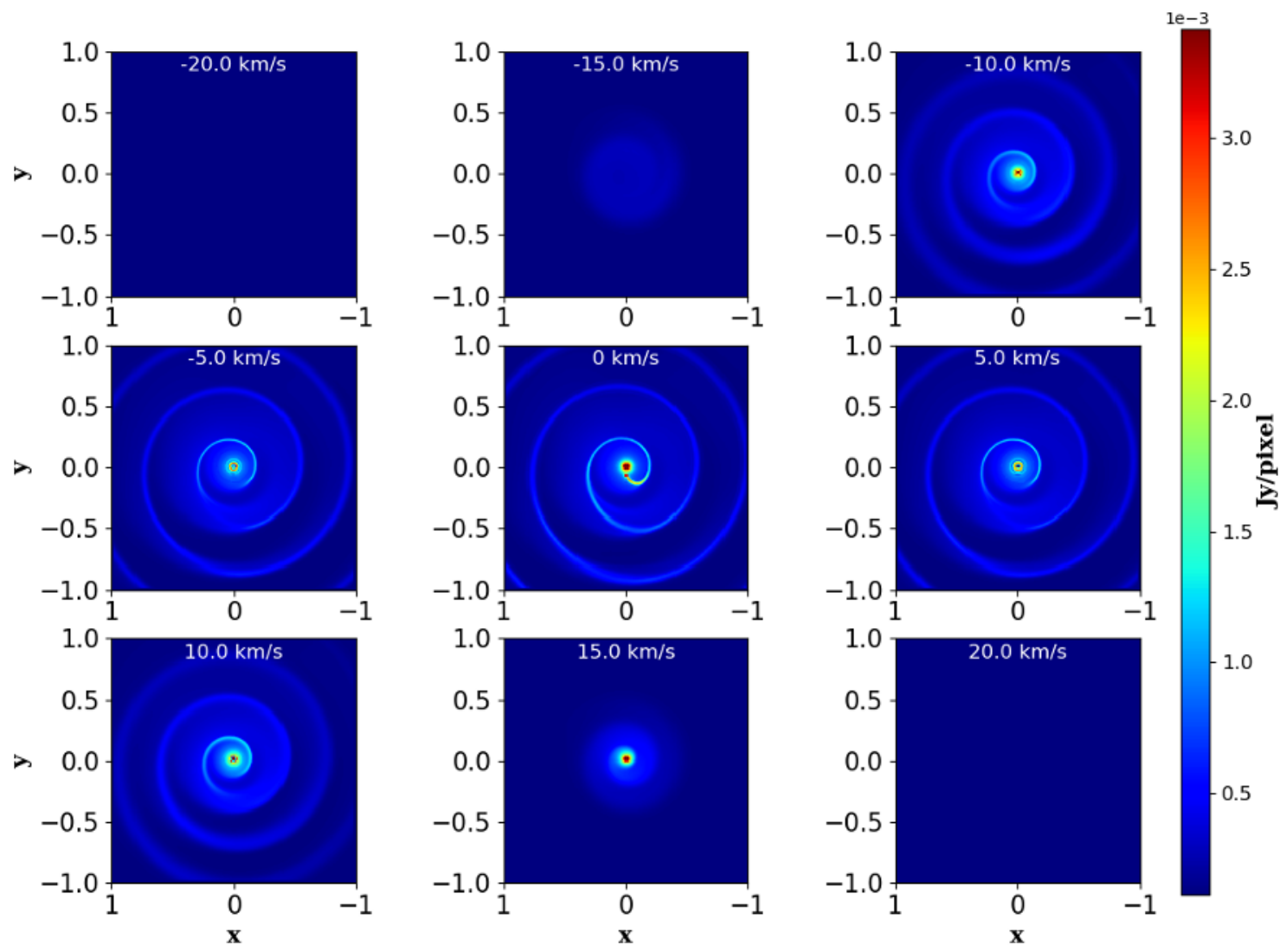

Fig. 4. Velocity channel maps of $\mathrm{HC}_{3} \mathrm{~N} J=5-4$ emission at viewing angle 0 degree for the case of binary companion moving in elliptical orbit with eccentricity $e=0.5$. 

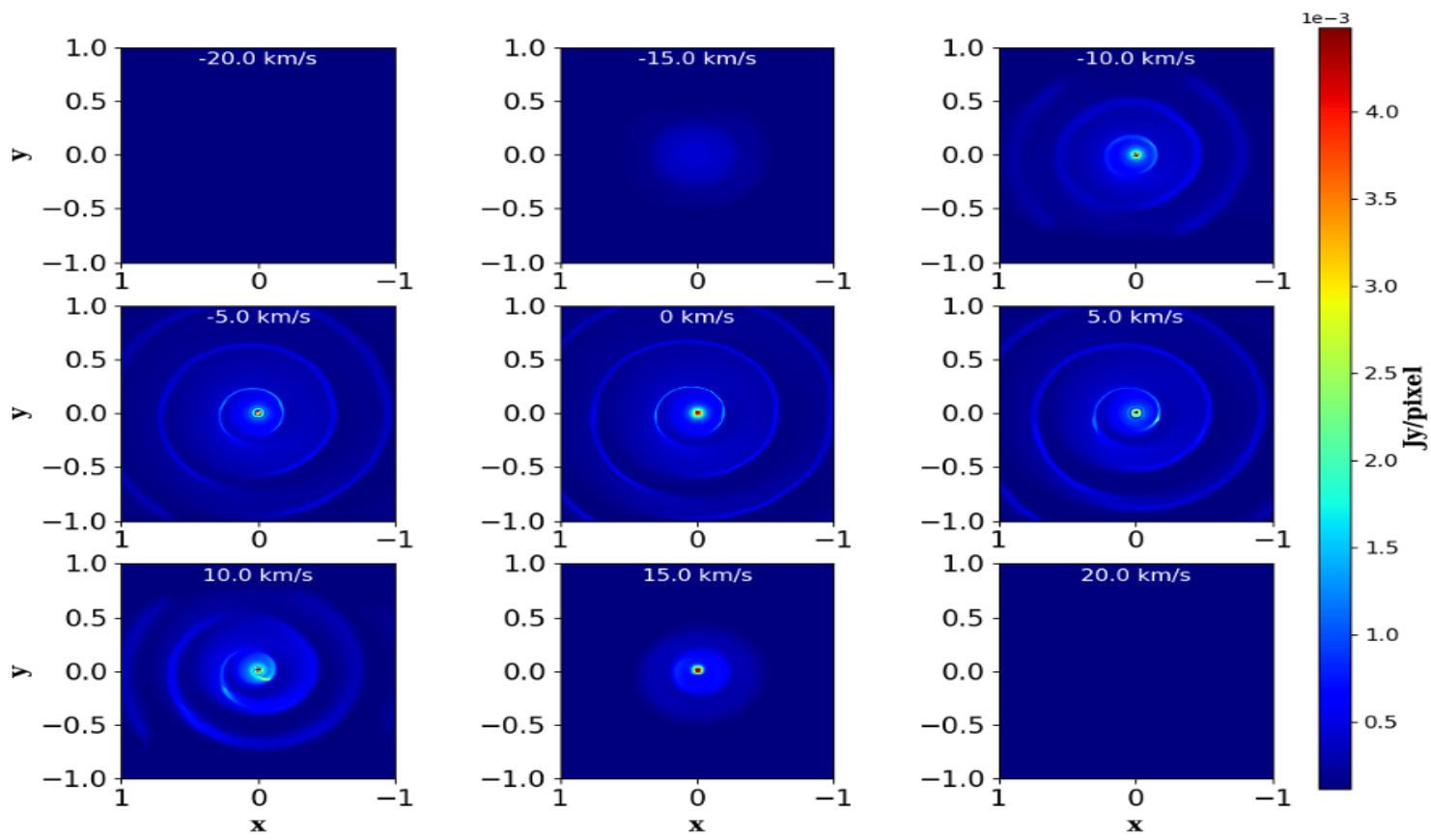

Fig. 5. Same as Fig. 4 but for viewing angle of 50 degrees.
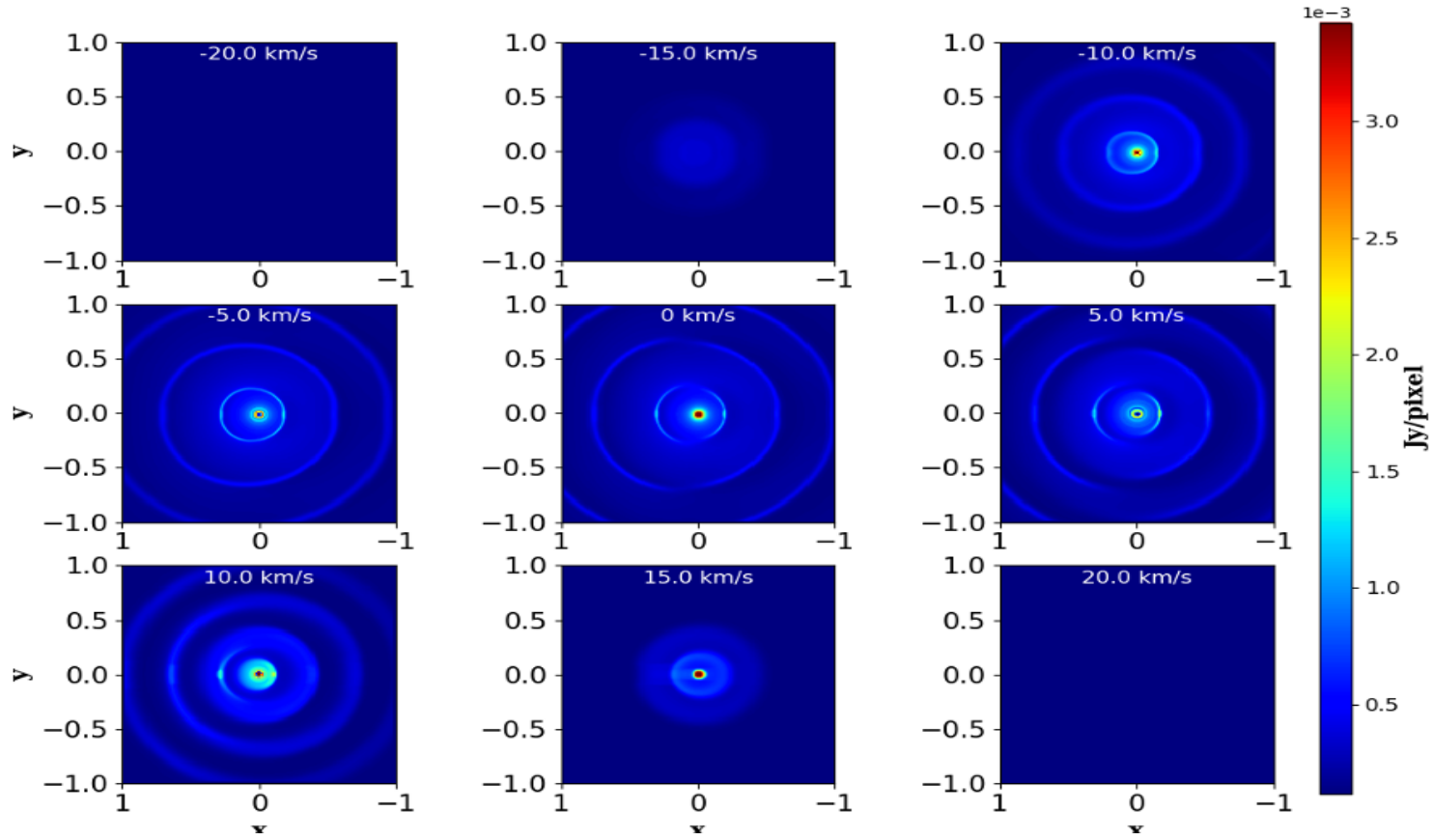

Fig. 6. Same as Fig. 4 but for viewing angle of 90 degrees. 

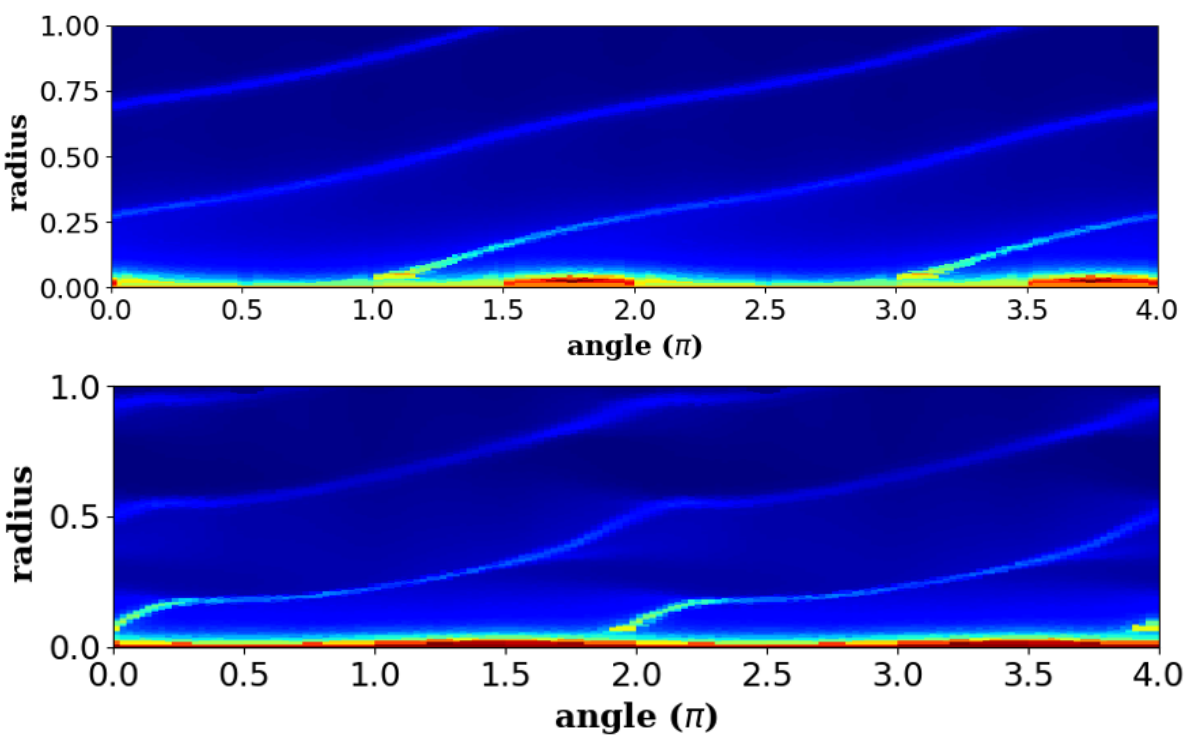

Fig. 7. The position - angle diagram of the $\mathrm{HC}_{3} \mathrm{~N} J=5-4$ line emission for the systemic velocity channel with the companion moving in (upper frame) circular orbit and (lower frame) elliptical orbit with $e=0.5$.

Because the molecular emissions encode both the spatial structure and kinematic information of different regions within the envelope, comparison of radiative transfer results incorporating realistic hydrodyamic simulations with high angular resolution interferometric observations could lead to a detailed reconstruction of the kinematics and physical conditions of the spiral pattern induced by the presence of a binary companion within the circumstellar envelopes. We hope to address this problem in a future publication.

\section{CONCLUSIONS}

In this paper we have presented the results of our radiative transfer calculations for the $\mathrm{HC}_{3} \mathrm{~N} J=5-4$ line based on the hydrodynamic simulations of the circumstellar envelopes in the presence of binary companion moving in different orbital configuration. We show that the molecular emission traces closely the spiral structure induced by the binary companion. In addition, we show that the morphology of the molecular emission depends on both the orbital configuration and the viewing angle producing different morphologies from spiral patterns to nearly concentric rings or arcs offset from the position of the AGB star. Comparison between high angular resolution observations and the predictions of our hydrodynamic and radiative transfer models will be useful for constraining the properties of circumstellar envelopes and the associated binary systems.

\section{ACKNOWLEDGEMENTS}

We acknowledge the financial support of the Vietnam Academy of Science and Technology (VAST) through project number NVCC.05.05/20-20. We would like to express our thanks to the Computing Center of VAST for support through special project on high performance computing. 


\section{REFERENCES}

[1] S. Hofner and H. Olofsson, Annu. Rev. Astron. Astrophys. 26 (2018) 92

[2] H. Kim, I. T. Hsieh, S. Y. Liu, R. E. Taam, Astrophys. J. 776 (2013) 86

[3] H. Kim, T. Alfonso, S. Y. Liu, R. Sahai, R. E. Taam, M. R. Morris, N. Hirano and I. T. Hsieh, Nat. Astron. 1 (2017) 60

[4] M. Maercker, S. Mohamed, W. H. T. Vlemmings, S. Ramstedt, M. A. T. Groenewegen, E. Humphreys, F. Kerschbaum, M. Lindqvist, H. Olofsson, C. Paladini, M. Wittkowski, I. De Gregorio-Monsalvo and L. A. Nyman, Nature 490 (2012) 232

[5] M. Morris, Publ. Astron. Soc. Pac. 99 (1987) 1115

[6] N. Mastrodemos and M. Morris, Astrophys. J. 523 (1999) 357

[7] H. Kim and R. E. Taam, Astrophys. J. 759 (2012) 59

[8] H. Kim, S. Y. Liu and R. E. Taam, Astrophys. J., Suppl. Ser. 243 (2019) 35

[9] S. Ramstedt, S. Mohamed, W.H.T. Vlemmings, T. Danilovich, M. Brunner, E. de Beck, E.M.L. Humphreys, M. Lindqvist, M. Maercker, H. Olofsson, F. Kerschbaum and G. Quintana-Lacaci, Astron. Astrophys. 605 (2017) A126

[10] Tran Ngoc Hung, Dinh Van Trung, Nguyen Thi Thanh Bao, Bui Van Hai and Pham Dong Bang, Comm. Phys. 29 (2019) 433

[11] A. Mignone, G. Bodo, S. Massaglia, T. Matsakos, O. Tesileanu, C. Zanni, A. Ferrari, Astrophys. J., Suppl. Ser. 170 (2007) 228

[12] Dinh-V-Trung and J. Lim, Astrophys. J. 701 (2009) 292

[13] P. Audinos, C. Kahane, R. Lucas, Astron. Astrophys. 287 (1994) L5

[14] M. Guelin, N. A. Patel, M. Bremer, J. Cernicharo, A. Castro-Carrizo, J. Pety, J. P. Fonfria, M. Agundez, M. Santander-Garcia, G. Quintana-Lacaci, L. Velilla Prieto, R. Blundell and P. Thaddeus, Astron. Astrophys. 610 (2018) A4

[15] P. J. Huggins and A. P. Healy, Astrophys. J. 304 (1986) 418

[16] Dinh-V-Trung and J. Lim, Astrophys. J. 678 (2008) 303 